\title{
Millisecond accuracy video display using OpenGL under Linux
}

\author{
NEIL STEWART \\ University of Warwick, Coventry, England
}

\begin{abstract}
To measure people's reaction times to the nearest millisecond, it is necessary to know exactly when a stimulus is displayed. This article describes how to display stimuli with millisecond accuracy on a normal CRT monitor, using a PC running Linux. A simple C program is presented to illustrate how this may be done within X Windows using the OpenGL rendering system. A test of this system is reported that demonstrates that stimuli may be consistently displayed with millisecond accuracy. An algorithm is presented that allows the exact time of stimulus presentation to be deduced, even if there are relatively large errors in measuring the display time.
\end{abstract}

In many psychology experiments, a stimulus is presented, and the latency of a participant's response is recorded. There are two key pieces of information that are required to determine a participant's reaction time: the time at which the stimulus was presented and the time at which the participant responded. I have described how to record responses with millisecond accuracy under Linux in Stewart (2006). The present article is concerned with the presenting of stimuli with millisecond accuracy.

\section{A Choice of Display Method}

A standard cathode ray tube (CRT) monitor works by scanning a beam of electrons over a phosphorus-coated screen. When the beam strikes the phosphorus, the phosphorus fluoresces. The beam scans each row of pixels from left to right, beginning with the top row and ending with the bottom row. The intensity of the beam is varied with location, to cause the phosphorus to fluoresce with different intensity, allowing a picture to be built up over a scan of the whole screen. The number of scans of the entire screen per second is called the refresh rate.

Recently, liquid crystal displays (LCDs) have been introduced. These work quite differently. When a voltage is applied to a liquid crystal, it changes its shape. Differentshaped crystals rotate the plane of polarized light by different extents. In an LCD, a layer of liquid crystals is sandwiched between two orthogonal polaroids. Unless the plane of the polarized light is rotated in between the two polaroids, it cannot pass through them. By varying the voltage applied to the liquid crystals in the sandwich, the degree of rotation of the plane of the polarized light varies, allowing more or less light to pass through the

I thank Dave Sleight for constructing the photodiode apparatus and James Adelman and Matthew Roberts for sharing their Linux knowledge. Correspondence concerning this article should be addressed to N. Stewart, Department of Psychology, University of Warwick, Coventry CV4 7AL, England (e-mail: neil.stewart@warwick.ac.uk).
LCD. By having a large array of liquid crystal segments, each controlled independently, the amount of light passing through different parts of the LCD can be controlled to create a picture. In thin film transistor (TFT) LCD panels, a transistor and capacitor assembly for each pixel is used to maintain the voltage across the liquid crystal between refreshes of the display.

Plant, Hammond, and Turner (2004) studied the performance of some CRT displays and some TFT LCD displays, by measuring the time for the display to become visible (as measured by a photodiode and oscilloscope apparatus). The CRTs tested reached full brightness in less than $1 \mathrm{msec}$. However, the brightness of the LCDs tested changed very slowly, taking $10-20 \mathrm{msec}$ for the display to reach full brightness (i.e., to warm up), depending on the display. Furthermore, the LCD displays took 5-20 msec for the image to fade to the background level of brightness on stimulus offset (i.e., to cool down). ${ }^{1}$ The point during the warm-up time at which an image is detectable by a human observer and, similarly, the point at which it is no longer visible during the cool-down time is likely to vary with environmental factors (e.g., ambient illumination or the brightness of other stimuli) and history (e.g., the brightness of recent stimuli). Thus, Plant et al.'s study shows that LCD TFT displays are not suitable for presenting stimuli with millisecond accuracy. Here, I will describe how to control a CRT display with millisecond accuracy in Linux.

\section{A Simple Linux Program ${ }^{2}$}

The most commonly used graphics system under Linux is $\mathrm{X}$ Windows. $\mathrm{X}$ Windows provides a graphical engine that is hardware independent and can be used across a large variety of operating systems, such as Mac OS X and Sun Solaris. Linux systems usually use a free implementation of the X Windows standard, called XFree86 (www .xfree86.org). The X Windows system performs windowing tasks, such as allocating new windows, moving them around, and deciding when they need to be redrawn. In this 
simple program, I have used the OpenGL graphics library to draw into a new window that fills the entire screen. OpenGL is also hardware independent and runs on many other operating systems. I used the Mesa3D implementation of the OpenGL standard (www.mesa3d.org). ${ }^{3}$

The complete $\mathrm{C}$ source code for the program is available from www.warwick.ac.uk/staff/Neil.Stewart/. The program first creates a blank, borderless window that fills the entire screen. This is done using $\mathrm{X}$ and OpenGL library functions. If you would like more information than is provided by the man pages for these functions, consult Nye (1992) and Woo, Neider, Davis, and Shreiner (1999). First, XOpenDisplay is used to open a connection to the $\mathrm{X}$ Server and obtain server display information. Second, $\mathrm{glXChooseVisual} \mathrm{is} \mathrm{used} \mathrm{to} \mathrm{select,} \mathrm{from} \mathrm{all} \mathrm{available} \mathrm{visu-}$ als on the display, the type of visual that best meets a minimum specification. Third, glXCreateContext is used to create a rendering context with direct access to the video card. Fourth, a new window that fills the entire screen and has no border is made using XCreateWindow. The attributes for this window are extracted from those of the root window. Fifth, to display or map the window to the screen, XMapWindow is called, followed by XIfEvent. XIfEvent waits for the window to be displayed before allowing execution to proceed.

So far, a new window has been created with an OpenGL rendering context that renders directly to the video card. Next, the details of the OpenGL system need to be specified. The rendering context is selected with glXMakeCurrent, and the coordinate system established with glViewport. OpenGL, which is a 3-D rendering system, can be set to 2-D by setting the model and projection transforms to the identity matrix (by calling glMatrixMode and glLoadIdentity) and calling glOrtho2D.

The final step is to hide the cursor. Unfortunately, to hide the cursor, $\mathrm{X}$ Windows requires the creation of a blank cursor, using XCreatePixmapCursor by passing a blank pixmap created with XCreateBitmapFromData as a parameter. The cursor is defined as the current cursor, using XDefineCursor. Together, all of these steps may seem rather lengthy, but they will work on any X Windows System with OpenGL.

From this point, things are much more simple. The rendering context that the program selects is a doublebuffered context. In double buffering, while the contents of one buffer are being displayed, drawing takes place on the other buffer. When a glXSwapBuffers call is made, the roles of the two buffers are reversed. The reversal is synchronized to the screen retrace, so that the buffers are swapped while the beam is moving from the bottom right corner at the end of a trace back to the top left corner for the beginning of the next. This means that if buffers are swapped every frame, there is one frame period (e.g., $10 \mathrm{msec}$ at $100-\mathrm{Hz}$ refresh rate) for drawing into a buffer. Because modern computers are very fast, it is possible to draw a lot in this time (cf. current computer games such as Doom 3 and Half-Life 2), depending on the processor and graphics card. There is no longer a need to buffer a really large number of frames in advance (in comparison with only 5 years ago; Graves \& Bradley, 1988a, 1988b; Myors, 1998, 1999; Tsai, 2001).

As an example, the following code fragment draws a red rectangle in the center of a green screen:

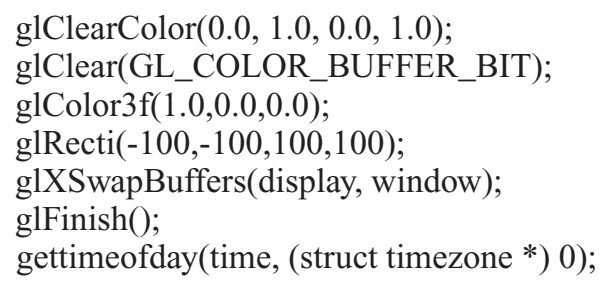

The clear color is set with glClearColor (which takes red, green, blue, and alpha values) before the screen is cleared by glClear. The drawing color is set to red by glColor $3 \mathrm{f}$ (which takes red, green, and blue values), and a rectangle is drawn with glRecti, with the bottom left corner at $-100,-100$ and the top right corner at 100,100 . The glXSwapBuffers call swaps the buffers, displaying the rectangle. The glFinish call ensures that all the commands are complete before it returns. Thus, the gettimeofday call sets the variable time to the time at which the tracing of the first frame containing the rectangle began.

\section{A Test of Millisecond Accuracy}

I conducted a simple test of the accuracy of the times recorded as above. I set up a simple loop to alternate the screen colors on every retrace of the screen between black and white and recorded the time after every call to glXSwapBuffers and glFinish. If the times are accurate, the intervals between times should be exactly the refresh period.

The system I ran these tests on was a dual-processor 1.4-MHz AMD Athlon with $256 \mathrm{MB}$ of RAM, with a PCI NVidia GeForce 2 MX with $32 \mathrm{MB}$ of video RAM. I used the Debian Woody GNU distribution with a 2.4.25-1-k7smp Linux kernel, XFree86 4.1.0 and the NVidia 1.0-5336 driver ${ }^{4}$ (and not the nv driver that comes with XFree86). The CRT monitor was a Sony CPD-G220 Color Trinitron running with a refresh rate of $85 \mathrm{~Hz}$. The following source code was used to sample the time of 10,000 consecutive refreshes:

$$
\begin{aligned}
\text { for(i } & =0 ; \mathrm{i}<10000 ; \mathrm{i}++)\{ \\
& \mathrm{c}=\text { double(i\%2); } \\
& \text { glClearColor(c, c, c, 1.0); } \\
& \text { glClear(GL_COLOR_BUFFER_BIT); } \\
& \text { glXSwapBuffers(display, window); } \\
& \text { glFinish(); } \\
& \text { gettimeofday(time[i], (struct timezone *) 0); }
\end{aligned}
$$

By alternating the color of the screen on alternate refreshes between black and white (using the variable $c$ ), a photodiode apparatus that was connected to the screen could detect the refreshes. During the test, no refreshes were missed (i.e., the screen color swapped every $1 / 85 \mathrm{sec}$ ).

For a vertical refresh rate of $85 \mathrm{~Hz}$, the period should be $11.765 \mathrm{msec}$. Figure 1 shows the differences between the consecutive times recorded in the array time. Only 12 intervals (about 1 in 1,000) fell outside the range of $\pm 0.05 \mathrm{msec}$ from the expected value of $11.765 \mathrm{msec}$. 


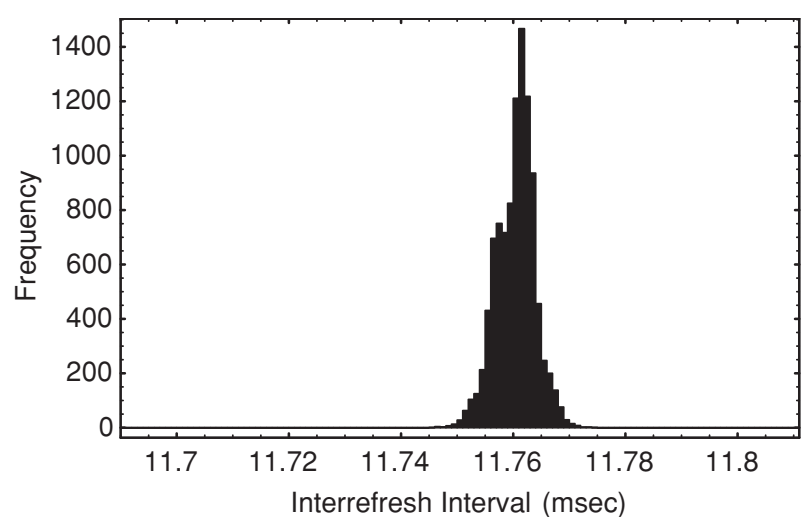

Figure 1. The distribution of interrefresh intervals measured by a gettimeofday call after a gIXSwapBuffers and a glFinish.

These cases occurred on the six occasions when there was a large gap between the glXSwapBuffers and glFinish calls and the gettimeofday call. The large gap caused the preceding interrefresh interval to be overestimated and the following interrefresh interval to be underestimated by the same amount. The largest error was $5 \mathrm{msec}$. The very rare errors of up to $5 \mathrm{msec}$ may not be of any concern, because they are so rare, but if they are, fortunately, they are very easy to correct, using the algorithm described below.

A second, more realistic test was also run. The duration of a white screen presented for $1,000 \mathrm{msec}$ (i.e., 85 retraces at an $85-\mathrm{Hz}$ refresh rate) was measured for each of 1,000 trials. The intertrial interval was also $1,000 \mathrm{msec}$, during which time a black screen was presented. The total duration of the test was about half an hour. Across the 1,000 trials, the minimum recorded display time was $999.734 \mathrm{msec}$, and the maximum was $999.800 \mathrm{msec}$. This gives a total range of only $0.066 \mathrm{msec}$ for the stimulus durations, which is an accuracy of better than one tenth of a millisecond. 5

\section{A Simple Correction}

For a monitor driven at $v \mathrm{~Hz}$, the times of the beginnings of the refresh must all be $1 / v$ sec apart. By taking advantage of this fact, the exact time of stimulus retraces can be deduced, providing that the error in recording them is small, in comparison with the refresh period $1 / v$. This model can be formalized in Equation 1:

$$
t_{i}=t_{0}+i / v
$$

where $t_{i}$ is the time (in seconds) of the $i$ th retrace and $i$ indexes the number of retraces since the first at time $t_{0}$. Suppose that one has recorded a series of retrace times $r_{j}$ (where $j$ indexes the number of recordings since $t_{0}$ ) and that there is some noise in the recording that varies from trial to trial, so that

$$
r_{j}=t_{i}+\varepsilon_{j},
$$

where $\varepsilon_{j}>0$ is the error in recording the time. From Equations 1 and $2, r_{j}-t_{0}=i / v+\varepsilon_{j}$. In other words, apart from the error in recording the time, $r_{j}-t_{0}$ must be an integer multiple of the refresh period $1 / v$. Under the assumption that $\varepsilon_{j}<1 / v$,

$$
i=\text { Quotient }\left(r_{j}-t_{0}, 1 / v\right),
$$

where the function Quotient[ ., . ] gives the integer part of the division of the first parameter by the second. That is, the retrace $i$ on which the $j$ th recording $r_{j}$ was made can be determined. Once $i$ is known, the exact time of the retrace $i$ is given by Equation 1 .

However, $t_{0}$ is not known exactly. But it can be estimated from the data. First, construct the error term

$$
\text { error }=\left[\operatorname{Mod}\left(r_{j}-t_{0}, 1 / v\right)\right]^{2},
$$

where the function $\operatorname{Mod}(.,$.$) returns the remainder after$ the first parameter is divided by the second. Error is the squared deviation between the recorded time $r_{j}$ and the first preceding modeled retrace time (the location of which is determined by $t_{0}$ ). Second, average this term over all of the refresh times recorded. Third, find the value of $t_{0}$ for which this sum is a minimum.

To test this algorithm, I have generated 1,000 trials of artificial refresh data, using Equations 1 and 2 as follows. The retraces on which recordings were made (i.e., the $i$ s) were generated by starting with a seed value of $i=0$ and generating each subsequent value by adding to the previous value an integer drawn at random from a uniform distribution of integers between 1 and 20. In this way, the retraces recorded were irregularly spaced, as they might be in an experiment in which the interstimulus intervals depend on a participant's response times. The noise $\varepsilon_{j}$ was modeled as a $\log$ normal distribution with a mean of $2 \mathrm{msec}$ and a standard deviation of $2 \mathrm{msec}$. This standard deviation is 20 times greater than the variability observed in the practical test above, and so this test is comparatively very tough. And $t_{0}$ was set to an arbitrary value of $325 \mathrm{msec}$. In this way, 1,000 simulated $r_{j}$ s were generated.

The average error (Equation 4) is plotted as a function of $t_{0}$ in Figure 2. The function is periodic and has a minimum every $1 / v \mathrm{sec}$. The minima immediately preceding $r_{0}$

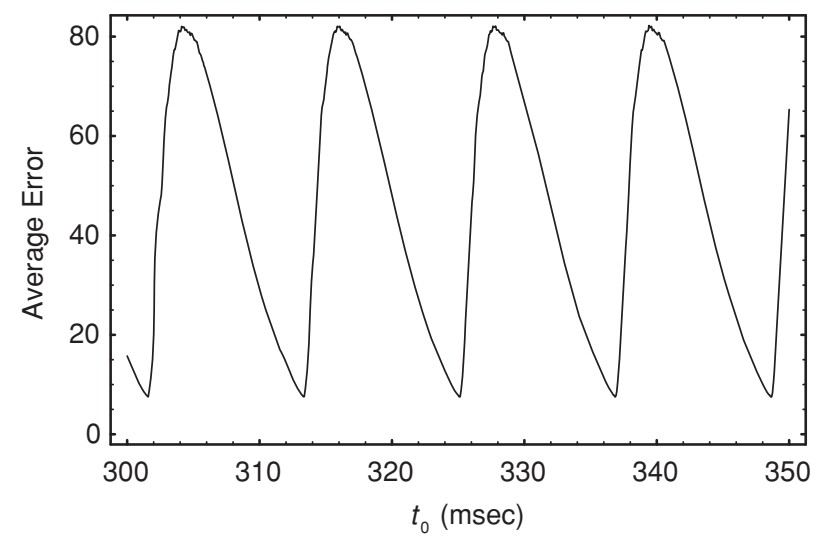

Figure 2. Model error as a function of $t_{0}$. 

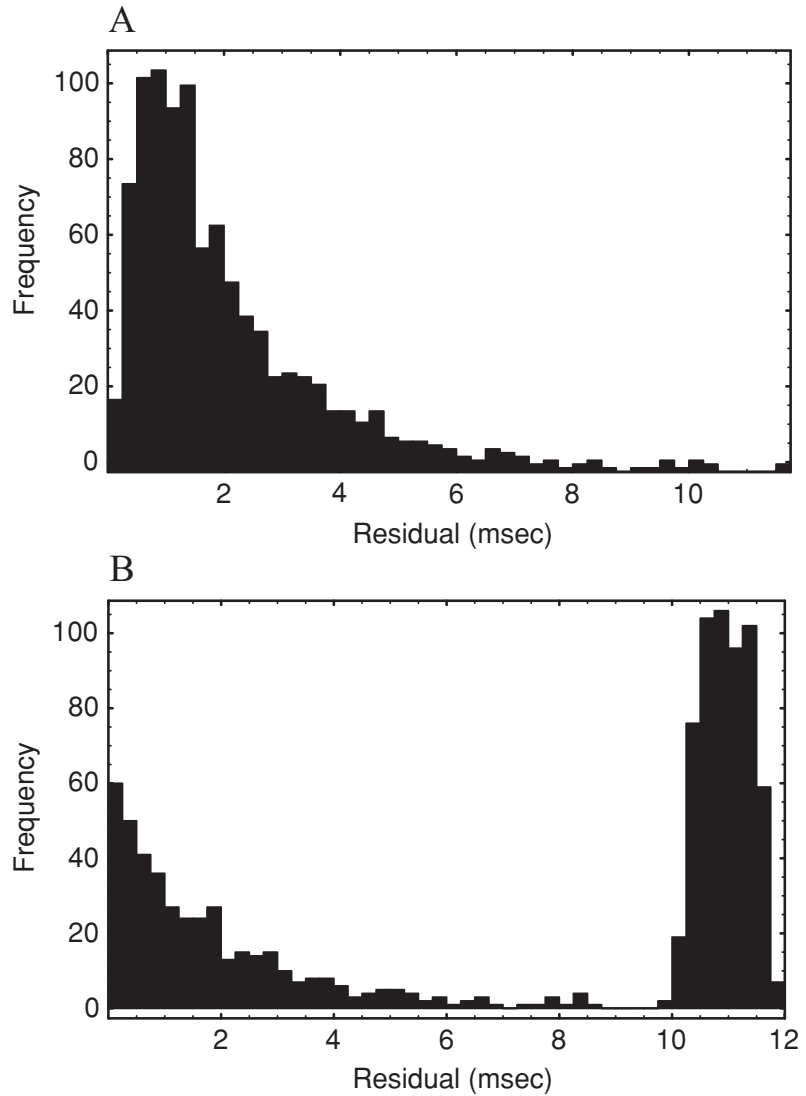

Figure 3. The distribution of residuals with $t_{0} \operatorname{correct}(\mathrm{A})$ and incorrect (B).

is $t_{0}$. Once $t_{0}$ is known, Equation 3 can be used to recover the numbers of the retraces (i.e., the $i$ s) upon which the recordings were made. Substituting these $i$ s into Equation 1 allows the exact retrace times to be calculated. As a check, the distribution of residuals $\left(r_{j}-t_{i}\right)$ can be plotted. Figure $3 \mathrm{~A}$ shows the distribution with $t_{0}=325$. However, if an incorrect value for $t_{0}$ is used, the distribution becomes bimodal. Figure 3B illustrates this occurring when $t_{0}=315$.

In summary, by taking advantage of the fact that retraces can occur only at certain predefined times, noisy timing data can be cleaned up, providing a much better estimate of the time at which a stimulus is displayed and eliminating trial-to-trial variability. Although the Linux system described above almost always performed very accurately, this method allows the very rare errors to be corrected.

\section{Conclusion}

In conclusion, a PC Linux system and a CRT monitor can be used to display stimuli with millisecond accuracy.

\section{REFERENCES}

Finney, S. A. (2001). Real-time data collection in Linux: A case study. Behavior Research Methods, Instruments, \& Computers, 33, 167173.

Graves, R. E., \& Bradley, R. (1988a). Millisecond timing on the IBM $\mathrm{PC} / \mathrm{XT} / \mathrm{AT}$ and PS/2: A review of the options and corrections for the Graves and Bradley algorithm. Behavior Research Methods, Instruments, \& Computers, 23, 377-379.

Graves, R. [E.], \& Bradley, R. (1988b). More on millisecond timing and tachistoscope applications for the IBM PC. Behavior Research Methods, Instruments, \& Computers, 20, 408-412.

MacInnes, W. J., \& TAYlor, T. L. (2001). Millisecond timing on PCs and Macs. Behavior Research Methods, Instruments, \& Computers, 33, 174-178.

Myors, B. (1998). The PC tachistoscope has 32 pages. Behavior Research Methods, Instruments, \& Computers, 30, 457-461.

Myors, B. (1999). The PC tachistoscope has 240 pages. Behavior Research Methods, Instruments, \& Computers, 31, 329-333.

Nye, A. (1992). Xlib programming manual (3rd ed.). Sebastopol, CA: O'Reilly.

Plant, R. P., Hammond, N., \& Turner, G. (2004). Self-validating presentation and response timing in cognitive paradigms: How and why? Behavior Research Methods, Instruments, \& Computers, 36, 291-303.

SteWART, N. (2006). A PC parallel port button box provides millisecond response time accuracy under Linux. Behavior Research Methods, 38, $170-173$.

TsaI, J.-L. (2001). A multichannel PC tachistoscope with high resolution and fast display change capability. Behavior Research Methods, Instruments, \& Computers, 33, 524-531.

Woo, M., Neider, J., Davis, T., \& Shreiner, D. (1999). OpenGL programming guide (3rd ed.). Reading, MA: Addison-Wesley.

\section{NOTES}

1. Plant et al. (2004) also found that there was a delay of up to $50 \mathrm{msec}$ between the time at which E-Prime software was scheduled to display the image and the time at which the image began to appear on the display.

2. For a multitasking operating system (e.g., Linux, Mac OS X, or MS Windows) to be able to function with millisecond accuracy, one must prevent the program from being interrupted and having its memory swapped to disc. An accurate timer is also required. Finney (2001; see also MacInnes \& Taylor, 2001; Stewart, 2006) describes how to ensure that a Linux program meets this standard.

3. The OpenGL utility toolkit (GLUT) can be used to simplify the process of initializing and opening new windows and controlling window events. In GLUT, buffers are swapped by calling glutSwapBuffers, which in turn calls glXSwapBuffers.

4. To ensure that buffers are swapped only during the vertical blanking period by glXSwapBuffers, the environment variable __GL_SYNC_TO_ VBLANK must be set to a nonzero value. It is easy to spot when buffer swapping is not synchronized, because the screen seems to "tear."

5 . The fact that the total display time was slightly less than $1,000 \mathrm{msec}$ indicates either that the actual monitor refresh rate was slightly faster than $85 \mathrm{~Hz}$ or that the computer's clock was running slightly slowly. The clock on the test computer loses about $2.6 \times 10^{-7} \mathrm{sec}$ per second, as measured by the network time protocol software (www.ntp.org).
(Manuscript received November 26, 2004; accepted for publication February 8, 2005.) 